Mathematical Modelling and AnAlysis

Volume 17 Number 2, April 2012, 141-160

http://dx.doi.org/10.3846/13926292.2012.655787

(c) Vilnius Gediminas Technical University, 2012
Publisher: Taylor\&Francis and VGTU

http://www.tandfonline.com/TMMA

Print ISSN: 1392-6292

Online ISSN: 1648-3510

\title{
Fairing Arc Spline and Designing by Using Cubic Bézier Spiral Segments
}

\author{
Zulfiqar $\mathrm{Habib}^{a}$ and Manabu Sakai ${ }^{b}$ \\ ${ }^{a}$ COMSATS Institute of Information Technology \\ Defense Road, Off Raiwind Road, Lahore, Pakistan \\ ${ }^{b}$ Kagoshima University \\ Koorimoto 1-21-35, 890-0065 Kagoshima, Japan \\ E-mail(corresp.): drzhabib@ciitlahore.edu.pk \\ E-mail: msakai@sci.kagoshima-u.ac.jp
}

Received March 1, 2011; revised December 19, 2011; published online April 1, 2012

\begin{abstract}
This paper considers how to smooth three kinds of $G^{1}$ biarc models, the C-, S-, and J-shaped transitions, by replacing their parts with spiral segments using a single cubic Bézier curve. Arc spline is smoothed to $G^{2}$ continuity. Use of a single curve rather than two has the benefit because designers and implementers have fewer entities to be concerned. Arc spline is planar, tangent continuous, piecewise curves made of circular arcs and straight line segments. It is important in manufacturing industries because of its use in the cutting paths for numerically controlled cutting machinery. Main contribution of this paper is to minimize the number of curvature extrema in cubic transition curves for further use in industrial applications such as non-holonomic robot path planning, highways or railways, and spur gear tooth designing.
\end{abstract}

Keywords: approximation, arc spline, smoothing problem, spiral, gear designing.

AMS Subject Classification: 65D05; 65D07; 65D10; 65D17; 65D18.

\section{Introduction}

Arc spline curves are made of circular arcs and straight line segments. They are $G^{1}$ continuous and are used to reduce the excessive number of data points with minimum alternation of the original profiles $[2,16,18,20,22,35]$. The curvature of an arc spline can be easily controlled and the offset curve of an arc spline is another spline. These are important for computer controlled cutting machines, for highway and railway designing as a natural curve along which to plan the center line of a rail/road or railway, for non-holonomic (car like) robot paths, and to design a spur gear tooth. However, arc spline is not as flexible and smooth as B-spline. On other side B-spline is a rather complicated and its curvature is difficult to control, for example, due to the prescribed bound on the maximum curvature. Offset of a B-spline is a non-rational curve [19, 20]. 
According to Farin [6], "A curve is fair (smooth) if its curvature plot is continuous and consists of only a few monotone pieces. Regions of monotone curvature are separated by points of extreme curvature. The number of curvature extrema of a fair curve should thus be small ". Since spiral transitions are without any internal curvature extrema [10], therefore the process of arc spline fairing can be accomplished by replacing their parts with spiral segments preserving curvature continuity.

Parametric cubic polynomial in Bézier form has been successfully used for spiral transitions between circles and straight lines [13, 14, 15]. Cubic curves, although visually pleasing, are not always helpful since they might have unwanted cusps, loops, up to two inflection points, and curvature extrema [21, 25, 30, 36]. Several Hermite-type and osculatory transition curves using cubic polynomials are presented in $[5,6,7,19,26,27,28,32]$.

The curvatures of $G^{1}$ arc splines are strictly controlled but such arcs are not easy to use, and are not flexible enough [36, 37, 38]. Li \& Meek in [20] have considered an application of a pair of conics to smooth a C-shaped curve or a cubic Bézier curve to smooth S- and J-shaped curves. Arc spline is smoothed to $G^{2}$ continuity but this increase in smoothness is at the cost of increase in the number of curvature extrema. In their proposed method, the maximum number of spiral pieces in a single segment could be six for the C-shaped transition and is five for the S- and J-shaped transitions, for larger angle between tangents at the end-points of transition curve.

In this paper, a stable and robust method is proposed for the replacement of all the above mentioned three types of $G^{1}$ biarcs with their corresponding $G^{2}$ transition curves having not more than one inflection point in their curvature, i.e., maximum three spiral segments in a single transition curve. These transitions are between two circular arcs tangent to each other or between a straight line and a circular arc tangent to each other. Walton \& Meek [29] and Habib \& Sakai [13] considered planar $G^{2}$ transition between two circles with a single fair cubic Bézier curve. They showed there is no curvature extremum in the case of an S-shaped transition, and there is a single curvature extremum in the case of a C-shaped transition. However, their method does not provide any solution for transition curves when circular arcs on both sides are tangent to each other. Such cases are discussed in [3] but without any analysis of number of curvature extrema in a C- or S-shaped transition curves. J-shaped case is presented in [33] but again there is no discussion on transition when straight line and circular arc are tangent to each other.

Keeping in view the above mentioned problems, main objectives of our paper are:

a) To extend, complete, and prove the analysis of $[14,20]$ for the replacement of $G^{1}$ biarcs with $G^{2}$ continuous spline having two or maximum three spiral segments by using a single cubic Bézier curve for all types of transition curves.

b) To modify the method of Habib \& Sakai [13] for the C- and S-shaped transitions, when both circular arcs are tangent to each other.

c) To find a method for the J-shaped transition curve, when the straight line and circular arc are tangent to each other.

d) To find sufficient conditions for just one curvature extremum in C and J- 
shaped transition curves, and maximum two curvature extrema in an S-shaped transition curve.

e) To design spur gears by using an S-shaped transition curve between two circles of same radii and tangent outside to each other with minimum possible number of curvature extrema, necessary to minimize friction between teeth.

\section{Notations and Conventions}

To aid concise writing of mathematical expressions, the symbols $\cdot$ and $\times$ are used to denote the usual inner product and signed $z$-component of the threedimensional cross-product of two vectors in the $x y$ plane, respectively, e.g.,

$$
\boldsymbol{a} \cdot \boldsymbol{b}=a_{x} b_{x}+a_{y} b_{y}=\|\boldsymbol{a}\|\|\boldsymbol{b}\| \cos \theta, \quad \boldsymbol{a} \times \boldsymbol{b}=a_{x} b_{y}-a_{y} b_{x}=\|\boldsymbol{a}\|\|\boldsymbol{b}\| \sin \theta,
$$

where $\theta$ is the counterclockwise angle from $\boldsymbol{a}$ to $\boldsymbol{b}$.

The signed curvature of a parametric curve $\boldsymbol{p}(t)$ in the plane is

$$
\kappa(t)=\boldsymbol{p}^{\prime}(t) \times \boldsymbol{p}^{\prime \prime}(t) /\left\|\boldsymbol{p}^{\prime}(t)\right\|^{3},
$$

when $\left\|\boldsymbol{p}^{\prime}(t)\right\|$ is non-zero. Positive curvature has the center of curvature on the left as one traverses the curve in the direction of increasing parameter. For non-zero curvature, the radius of curvature, positive by convention, is $1 /|\kappa(t)|$.

The derivative $\kappa^{\prime}(t)$ of the curvature in (2.1) yields

$$
\kappa^{\prime}(t)=\phi(t) /\left\|\boldsymbol{p}^{\prime}(t)\right\|^{5}
$$

where $\phi(t)=\left\|\boldsymbol{p}^{\prime}(t)\right\|^{2} \frac{\mathrm{d}}{\mathrm{d} t}\left\{\boldsymbol{p}^{\prime}(t) \times \boldsymbol{p}^{\prime \prime}(t)\right\}-3\left\{\boldsymbol{p}^{\prime}(t) \times \boldsymbol{p}^{\prime \prime}(t)\right\}\left\{\boldsymbol{p}^{\prime}(t) \cdot \boldsymbol{p}^{\prime \prime}(t)\right\}$.

A curve is $G^{1}$ continuous if it is continuous and has a continuous unit tangent vector. A curve is $G^{2}$ continuous if is $G^{1}$ continuous and it has a continuous curvature. $G^{2}$ continuous curves are considered smoother than $G^{1}$ continuous ones.

Descartes rule of signs [17, pp. 439-443] is a technique for determining the number of positive or negative real roots of a polynomial. The term 'spiral' refers to a curved line segment whose curvature varies monotonically with constant sign. Based on Kneser's theorem [9], any circle of curvature of a spiral encloses all the smaller circles of curvature and is enclosed by all larger circles of curvature. So we cannot find the transition curve with a single spiral segment when the smaller circle is not completely inside the larger circle.

\section{Description of Method}

The arc spline is a planar $G^{1}$ continuous curve made of circular arcs and straight-line segments. The process of smoothing arc spline will be done by replacing the following three types of transitions:

C-shaped transition between two circular arcs having same signs of curvatures. 
S-shaped transition between two circular arcs having opposite signs of curvatures.

J-shaped transition between a straight-line segment and a circular arc.

A single cubic Bézier curve in parametric form will be used for all the above mentioned three types of $G^{2}$ continuous transition curves.

Let $\boldsymbol{C}_{0}$ and $\boldsymbol{C}_{1}$ be the centers of larger circle $\Omega_{0}$ and smaller circle $\Omega_{1}$, with radii $r_{0}, r_{1}$, respectively. The problem of finding a fair parametric transition curve from larger circle (for C- and S-shaped) or straight-line (for J-shaped) to smaller circle can be solved in Hermite-like manner, i.e., for given starting point, tangent and curvature at start point, and curvature at end point (end point and tangent vector at end point are restricted to the given circle $\Omega_{1}$ ). For C- and S-shaped transitions, radii of both circles should satisfy the condition $\mu>1$ and $\mu \geq 1$, respectively, where $\mu=\sqrt{r_{0} / r_{1}}$. The case $\mu<1$ is handled by reversing the biarc, converting the condition into $\mu>1$. For J-shaped transition, radius $r_{1}$ of circle $\Omega_{1}$ is given. C-, and S-shaped transition curves from circle to circle, and J-shaped transition curve from straight line to circle, are shown in normalized form in Figures 1, 3, and 5, respectively. In these figures, the point of contact of both circles, or straight-line and circle is shown as small circle.

Since the smaller circle $\Omega_{1}$ does not lie completely inside the larger circle $\Omega_{0}$, Kneser's theorem implies that there must be at least one internal curvature extremum in a transition curve. We obtain sufficient conditions for one or maximum two curvature extrema by use of Descartes rule of signs on the number of the positive roots of the derivative of curvature. For ease of presentation, we describe the biarc model in a local coordinate system. In practical implementation of smoothing arc splines, a world coordinate system is required. The conversion between local and world coordinate systems is a simple matter of transformation.

A cubic Bézier curve $\boldsymbol{z}(t)=(x(t), y(t))$, has eight degrees of freedom in the interval $t \in[0,1]$, and is represented as

$$
\boldsymbol{z}(t)=(1-t)^{3} \boldsymbol{p}_{0}+3 t(1-t)^{2} \boldsymbol{p}_{1}+3 t^{2}(1-t) \boldsymbol{p}_{2}+t^{3} \boldsymbol{p}_{3}
$$

where $\boldsymbol{p}_{i}, i=0, \ldots, 3$, are the four distinct control points. Without any loss of generality we can translate, rotate and, if necessary, reflect the shape to normalize it as shown in Figures 1, 3, and 5, so that the given starting point $\boldsymbol{p}_{0}$ is at the origin, $\boldsymbol{p}_{1}$ is on the positive $x$-axis, the given center $\boldsymbol{C}_{0}\left(=\left(0, r_{0}\right)\right)$ of the larger circle (for $\mathrm{C}$ - and $\mathrm{S}$-shaped) is on the positive $y$-axis, the ending point $\boldsymbol{p}_{3}$ is above the $x$-axis. Let the control polygon in (3.1) be represented by

$$
\{g, h, k\}=\left\{\left\|\boldsymbol{p}_{0} \boldsymbol{p}_{1}\right\|,\left\|\boldsymbol{p}_{1} \boldsymbol{p}_{2}\right\|,\left\|\boldsymbol{p}_{2} \boldsymbol{p}_{3}\right\|\right\} .
$$

In normalized form of cubic Bézier polygon, segment $h$ in $\mathrm{C}$ and S-shaped cases is making angle $\theta$ and segment $k$ in J-shaped case is making angle $2 \theta$ with the positive $x$-axis, where $\theta \in(0, \pi / 2)$. As will be seen later, angle $\theta$ is used as a free parameter providing some more freedom to the user in addition to comfortable range of other parameters for fair transition curves. To simplify 
the analysis, most of the expressions in this paper are written in terms of $u$ $\left(=\tan ^{2} \theta\right.$ ), instead of angle $\theta$. Other important parameters used to solve the problem are mentioned in the algorithm in Section 4.

The steps of our analysis are to derive the conditions for a single or maximum two curvature extrema in a transition curve. The results are expressed as theorems. The three cases of C, S, and J-shaped transitions are then considered separately in the following sections.

\subsection{C-shaped transition curve}

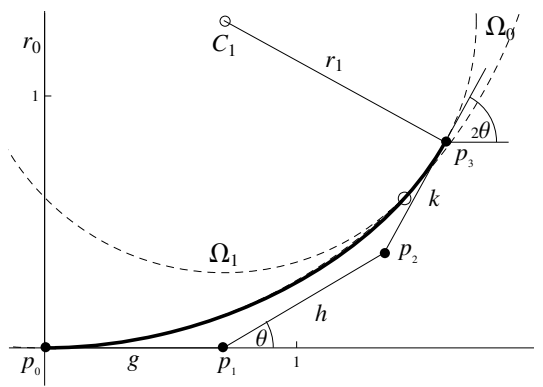

Figure 1. A C-shaped transition curve in normalized form.

Here we consider the case of C-shaped transition curve $\boldsymbol{z}(t)$ of the form (3.1) between two circles tangent inside to each other. Since one circle is not completely inside the other circle, the transition curve must has at least one interior curvature extremum. We show that the transition curve does not has more than one curvature extremum, i.e., it is composed with two spiral segments.

We assume, the segment $h$ is making an angle $\theta$ with segment $k$, i.e., angle between tangent vectors $\boldsymbol{z}^{\prime}(0)$ and $\boldsymbol{z}^{\prime}(1)$ of transition curve is in $(0, \pi)$, as shown in Figure 1. Recall that $\boldsymbol{p}_{1}-\boldsymbol{p}_{0}$ is parallel to the $x$-axis, i.e., the tangent vector $\boldsymbol{z}^{\prime}(0)$ is parallel to $(1,0)$. We summarize the above discussion as

$$
\begin{gathered}
\boldsymbol{p}_{0}=(0,0), \quad \boldsymbol{p}_{1}=(g, 0), \\
\boldsymbol{p}_{2}=\boldsymbol{p}_{1}+h(\cos \theta, \sin \theta), \quad \boldsymbol{p}_{3}=\boldsymbol{p}_{2}+k(\cos 2 \theta, \sin 2 \theta) .
\end{gathered}
$$

To simplify the analysis, again let $h=p^{2} r_{1}$. Then the end-points curvature conditions $(\kappa(0), \kappa(1))=\left(1 / r_{0}, 1 / r_{1}\right)$, yield

$$
(g, k)=p r_{1} \sqrt{2 \sin \theta / 3}(\mu, 1) .
$$

Therefore, from (3.1)-(3.3), we have a family of cubic transition curves

$$
\begin{aligned}
& x(t)=\frac{2 m r_{1} t \mu}{3}\left\{\left(3-3(1-m) t+(1-2 m) t^{2}\right) \mu+t^{2} \cos 2 \theta\right\} \tan \theta, \\
& y(t)=\frac{2 m r_{1} t^{2} \mu}{3}\left\{2 t \cos ^{2} \theta+m(3-2 t) \mu\right\} \tan ^{2} \theta
\end{aligned}
$$


obtained by introducing a parameter $m$ and choosing

$$
p=m \mu \sec \theta \sqrt{2 \sin \theta / 3} .
$$

For later use, to find sufficient conditions for the single curvature extremum in a C-shaped transition curve, the derivative of curvature of function (3.1) is required which is given by $(2.2)$. It is calculated for $t=s /(1+s)$. Parameter $s$ is introduced to simplify the analysis of expressions in derivative of curvature by removing the upper bound of $t: 0 \leq t \leq 1 \Rightarrow s \geq 0$. Now derivative of curvature can be represented as

$$
\phi(s)=\frac{64 m^{4} r_{1}^{4} u^{5 / 2} \mu^{5}}{(1+s)^{5}(1+u)^{2}} \sum_{i=0}^{5} c_{i} s^{i}
$$

where

$$
\begin{aligned}
& c_{0}=(1+u) \mu^{2}\left\{1+2 m(1+u) \mu-3 m^{2}(1+u) \mu\right\}, \\
& c_{1}=(1+u) \mu^{2}\left\{5-4 m(1-u)+5 m^{2}(1+u) \mu-6 m^{3}(1+u)^{2} \mu\right\}, \\
& c_{2}=2 \mu\left[2(u-1)-m(1+u)\{-9-u+8 m(1+u)\} \mu+m^{3}(1+u)^{3} \mu^{3}\right], \\
& c_{3}=2 \mu\left[2(1-u)-m\left\{9+10 u+u^{2}-m(1+u)^{2}(8-m+m u) \mu\right\}\right], \\
& c_{4}=(1+u)[-5+m \mu\{4-4 u+m(1+u)(-5+6 m(1+u) \mu)\}], \\
& c_{5}=(1+u)\{-1+m(1+u)(-2+3 m \mu)\} .
\end{aligned}
$$

In addition to the above mentioned conditions, we require that the larger circle $\Omega_{0}$ touches inside the osculating circle $\Omega_{1}$, i.e.,

$$
\left\|\boldsymbol{C}_{0}-\boldsymbol{C}_{1}\right\|=r_{0}-r_{1},
$$

where centers of both circles are $\boldsymbol{C}_{0}=\left(0, r_{0}\right), \boldsymbol{C}_{1}=\boldsymbol{z}(1)-r_{1}(\sin 2 \theta,-\cos 2 \theta)$, respectively.

Depending on either parameter $m$ or an angle $\theta$ is given, our method is divided in the following two cases.

Case 1. Parameter $m$ is given. In this case an angle $\theta$ needs to be calculated satisfying the condition of tangent circles in (3.7), giving a quadratic equation in $u$ as

$$
\begin{aligned}
& m^{4} \mu^{2} u^{2}-2 m^{2}\left\{1+(1-m) \mu+\left(1-m-m^{2}\right) \mu^{2}\right\} u \\
& \quad+9-6 m-2 m^{2}-2 m\left(3-m-m^{2}\right) \mu-m^{2}\left(2-2 m-m^{2}\right) \mu^{2}=0,
\end{aligned}
$$

where the solution of (3.8) for $u$ with $D=2 \mu+\{1+(1-m) \mu\}^{2}(>0)$ is

$$
u=\frac{1+(1-m) \mu+\left(1-m-m^{2}\right) \mu^{2} \pm(\mu-1) \sqrt{D}}{m^{2} \mu^{2}} .
$$

The $G^{2}$ transition curve exists if the larger solution of (3.9) is positive, leading to the conditions

$$
1<\mu<\frac{(1+\sqrt{3})\left(3-m^{2}\right)-(2 \sqrt{3}+1) m}{m\left(m^{2}+2 m-2\right)}, \quad-1+\sqrt{3}<m<1 .
$$


The following remark is required since the circular arc to be replaced by the transition curves are not usually the whole circles, i.e., just the portion of the circles.

Remark 1. The distance of end point $\boldsymbol{p}_{3}$ of transition curve from its start point $\boldsymbol{p}_{0}$ (i.e., origin) is given by

$$
\begin{aligned}
\|\boldsymbol{z}(1)\|= & \frac{2}{3} \\
& m r_{1} \mu \sec \theta \tan \theta \\
& \times \sqrt{m^{2} \mu^{2}+\left((1-\mu)^{2}+2 m \mu(1+\mu)\right) \cos ^{2} \theta+4 \mu \cos ^{4} \theta} .
\end{aligned}
$$

We can see that the transition curve reduces to the common point of the two circles as $\mu$ tends to the upper limit by (3.10) or $\theta$ tends to 0 .

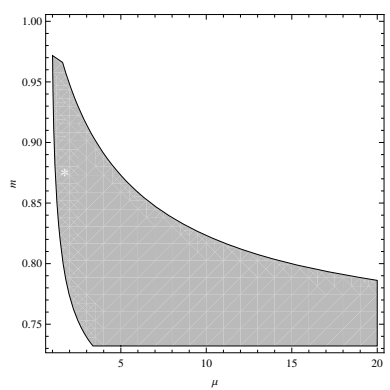

(a) Case 1: $m$ is given

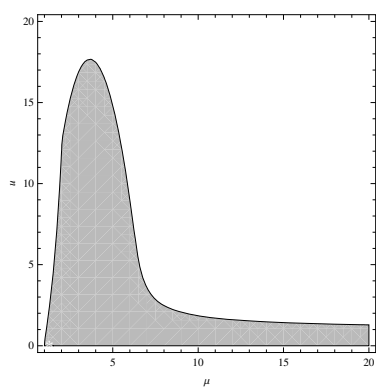

(b) Case 2: $\theta$ is given

Figure 2. Regions for single curvature extremum for a C-shaped transition curve.

From (3.6), the Descartes rule of signs gives the sufficient condition for the single curvature extremum as

$$
\min \left[c_{5}, c_{4}, c_{3}, c_{2},-c_{0}\right] \geq 0 .
$$

Then the following theorem guarantees the fairness of the desired single Cshaped transition curve for $(\mu, m)$ belonging to the gray region in Figure 2(a).

Theorem 1. The $G^{2}$ transition curve $\boldsymbol{z}(t)$ of the form (3.4) defined by (3.1) with given parameter $m$, between two given circles tangent inside to each other, exists and has exactly one curvature extremum for $(\mu, m)$ limited by (3.10) and (3.12).

Proof. Existence of solution is ensured from condition in (3.10) and the guarantee of single interior curvature extremum is given by (3.12).

Case 2. Angle $\theta$ is given. Angle $\theta$ is given in this case and therefore value of parameter $m$ is required. From (3.7), we have two positive real solutions

$$
\begin{aligned}
m= & \frac{1}{2(1+u) \mu}[-1 \pm \sqrt{3+2 u}-(1+\sqrt{3+2 u}) \mu \\
& \left.+\sqrt{2(1+u)\{2-\sqrt{3+2 u}+(2+\sqrt{3+2 u}) \mu\}+u\left(1+4 \mu+\mu^{2}\right)}\right] .
\end{aligned}
$$


Smaller solution of $m$ is giving us a more flexible sufficient condition than its larger one (shown as gray region in Figure 2(b)) for the single curvature extremum by applying Descartes rule of signs on (3.6) as

$$
\min \left[-c_{5}, c_{3}, c_{2}, c_{1}, c_{0}\right] \geq 0 \text {. }
$$

The following theorem guarantees the fairness of the desired single C-shaped transition curve for $(\mu, u)$ belonging to the gray region in Figure 2(b).

Theorem 2. The $G^{2}$ transition curve $\boldsymbol{z}(t)$ of the form (3.4) defined by (3.1) with given angle $\theta$, between two given circles tangent inside to each other, exists and has exactly one curvature extremum for $(\mu, u)$ limited by (3.14).

Proof. Existence of solution and the guarantee of single interior curvature extremum are ensured from (3.13) and condition in (3.14).

\subsection{S-shaped transition curve}

Here we consider an S-shaped transition curve $\boldsymbol{z}(t)$ of the form (3.1). We assume, segment $k$ is making an angle $\theta$ with segment $h$, i.e., the tangent vector $\boldsymbol{z}^{\prime}(0)$ of transition curve is parallel to the tangent vector $\boldsymbol{z}^{\prime}(1)$, and therefore both tangents are parallel to $x$-axis in the normalized form of control polygon of Bézier curve. It is shown in Figure 3.

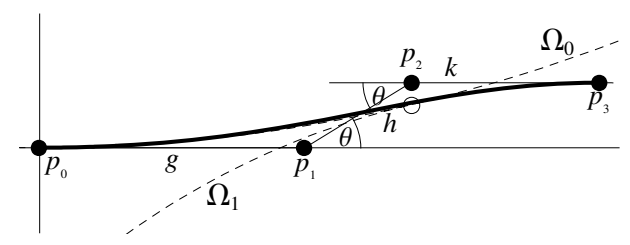

Figure 3. An S-shaped transition curve in normalized form.

Above discussion concludes the following results.

$$
\begin{gathered}
\boldsymbol{p}_{0}=(0,0), \quad \boldsymbol{p}_{1}=(g, 0), \\
\boldsymbol{p}_{2}=\boldsymbol{p}_{1}+h(\cos \theta, \sin \theta), \quad \boldsymbol{p}_{3}=\boldsymbol{p}_{2}+(k, 0) .
\end{gathered}
$$

To simplify the analysis, we assume $h=p^{2} r_{1}$. The curvature end conditions $(\kappa(0), \kappa(1))=\left(1 / r_{0},-1 / r_{1}\right)$, give us

$$
(g, k)=p r_{1} \sqrt{2 \sin \theta / 3}(\mu, 1) .
$$

Therefore from (3.1), we have a family of S-shaped transition curves

$$
\begin{aligned}
& x(t)=\frac{4}{27} m r_{1} t \mu\left\{9 \mu-3(3-2 m) t \mu+t^{2}(3+(3-4 m) \mu)\right\} \sqrt{u}, \\
& y(t)=\frac{8}{27} m^{2} r_{1} t^{2}(3-2 t) \mu^{2} u
\end{aligned}
$$


with a parameter $m$, obtained by introducing the constraints

$$
p=m \mu \sec \theta \sqrt{8 \sin \theta / 27} .
$$

Here the curvature of the transition curve should change sign to allow an inflection point in S-shaped transition curve. To find sufficient conditions for the single or double curvature extrema in an S-shaped transition curve, the derivative of curvature of function (3.1) is required for later use. It is obtained from (2.2) for $t=s /(1+s), s \geq 0$, and is given as

$$
\phi(s)=-\frac{4096 m^{5} r_{1}^{4} u^{5 / 2} \mu^{5}}{2187(1+s)^{5}} \sum_{i=0}^{5} s_{i} s^{i}
$$

where

$$
\begin{aligned}
& s_{0}=9 \mu^{3}(-1+m), \quad s_{1}=3 \mu^{2}\left\{6-5 m \mu+4 m^{2}(1+u) \mu\right\}, \\
& s_{2}=\mu^{2}\left\{9+30 m-4 m^{2}(1+u) \mu\right\}, \quad s_{3}=\mu\left\{9+30 m \mu-4 m^{2}(1+u) \mu\right\}, \\
& s_{4}=3 \mu\left\{6-5 m+4 m^{2}(1+u) \mu\right\}, \quad s_{5}=-9+9 m \mu .
\end{aligned}
$$

With all the above mentioned conditions, we also assume that the larger circle $\Omega_{0}$ touches outside the osculating circle $\Omega_{1}$, i.e.,

$$
\left\|\boldsymbol{C}_{0}-\boldsymbol{C}_{1}\right\|=r_{0}+r_{1}
$$

where centers of both circles are $C_{0}=\left(0, r_{0}\right), C_{1}=\boldsymbol{z}(1)-r_{1}(0,1)$, respectively.

Again depending on either parameter $m$ or an angle $\theta$ is given, the method for S-shaped transition curve is also divided in the following two cases.

Case 1. Parameter $m$ is given. In this case an angle $\theta$ needs to be calculated satisfying the condition of tangent circles in (3.20), giving

$$
u=\frac{9\left(1-\mu+\mu^{2}\right)-6 m \mu(1+\mu)-2 m^{2} \mu^{2}}{2 m^{2} \mu^{2}},
$$

where the positive right hand side of above expression requires

$$
m<\frac{3\left(1-\mu+\mu^{2}\right)}{\left.\mu\left\{1+\mu+\sqrt{3\left(1+\mu^{2}\right.}\right)\right\}} .
$$

Remark 2. The distance of end point $\boldsymbol{p}_{3}$ of transition curve from its start point $\boldsymbol{p}_{0}$ (i.e., origin) is given by

$$
\|\boldsymbol{z}(1)\|=\frac{4 m r_{1} \mu \tan \theta}{27} \sqrt{\{3+(3+2 m) \mu\}^{2}+4 m^{2} \mu^{2} \tan ^{2} \theta} .
$$

It shows that the transition curve reduces to the common point of the two circles as parameter $m$ tends to the upper limit by (3.22) or angle $\theta$ tends to 0 . 
Once again Descartes rule of signs on (3.19) gives the sufficient condition for the single curvature extremum as

$$
\min \left[s_{5}, s_{4}, s_{3}, s_{2},-s_{0}\right] \geq 0 \quad \text { or } \quad \min \left[s_{5}, s_{4}, s_{3},-s_{1},-s_{0}\right] \geq 0 .
$$

It is covering a large range of $\mu$ and is shown as light gray shade in Figure 4(a). For the remaining small range of $\mu$, the Descartes rule of sign suggests the possibility of two curvature extrema given by the condition

$$
\min \left[-s_{5}, s_{4}, s_{3}, s_{2},-s_{0}\right] \geq 0 .
$$

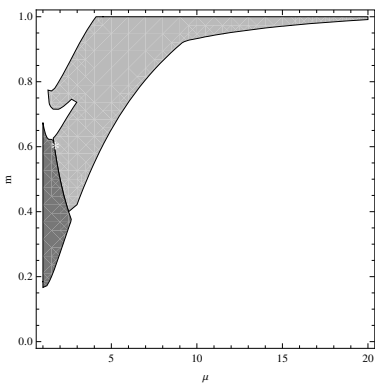

(a) Case 1: $m$ is given

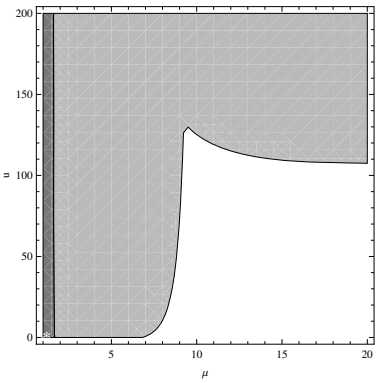

(b) Case 2: $\theta$ is given

Figure 4. Regions for single (light gray) or double (dark gray) curvature extrema for an S-shaped transition curve.

The following theorem guarantees the fairness of the desired S-shaped transition curve for $(\mu, m)$ belonging to the light or dark gray region in Figure 4(a).

Theorem 3. The $G^{2}$ transition curve $\boldsymbol{z}(t)$ of the form (3.17) defined by (3.1) with given parameter $m$ limited by (3.22), between two given circles tangent outside to each other, exists and has single or double curvature extrema for $(\mu, m)$ if conditions in (3.24) or (3.25) satisfied, respectively.

Proof. Existence of solution is ensured with condition in (3.22) and the guarantee of single or double interior curvature extrema is given by (3.24) or (3.25), respectively.

Case 2. Angle $\theta$ is given. In this case, angle $\theta$ is given and therefore the positive real value of parameter $m$ is obtained from (3.20) as

$$
m=\frac{3\left(-1-\mu+\sqrt{3\left(1+\mu^{2}\right)+2 u\left(1-\mu+\mu^{2}\right)}\right)}{2(1+u) \mu} .
$$

giving us the sufficient condition for the single curvature extremum by applying Descartes rule of signs on (3.19) as expressed in (3.24) covering a large range of $\mu$ and is shown as light gray shade in Figure 4(b). For the remaining small range of $\mu$, the Descartes rule of sign suggests the possibility of two curvature extrema given by the condition in (3.25). 
The following theorem guarantees the fairness of the desired single S-shaped transition curve for $(\mu, u)$ belonging to the gray region in Figure $4(\mathrm{~b})$.

Theorem 4. The $G^{2}$ transition curve $\boldsymbol{z}(t)$ of the form (3.17) defined by (3.1) with given angle $\theta$, between two given circles tangent outside to each other, exists and has single or double curvature extrema for $(\mu, u)$ if conditions in (3.24) or (3.25) are satisfied, respectively.

Proof. The proof is immediately follows from the above discussion.

Corollary 1. An S-shaped transition between two circles tangent outside to each other and of same radii (i.e., $\mu=1$ ) does not has less than two curvature extrema.

Proof. Its proof is followed from Descartes rule of signs and Kneser's theorem.

\subsection{J-shaped transition curve}

In this section, we consider a J-shaped transition curve $\boldsymbol{z}(t)$ of the form (3.1) and show that it does not has more than one curvature extremum, i.e., it is composed with two spiral segments. Recall that segment $g$ is parallel to $x$ axis in the normalized form of control polygon of J-shaped Bézier curve. We assume, segment $h$ is also parallel to $x$-axis and segment $k$ is making an angle $2 \theta$ with $x$-axis, i.e., angle between tangent vectors $\boldsymbol{z}^{\prime}(0)$ and $\boldsymbol{z}^{\prime}(1)$ of transition curve is in $(0, \pi)$. It is shown in Figure 5.

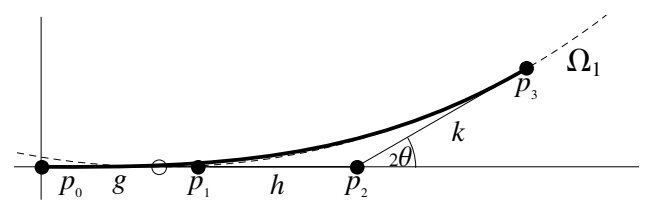

Figure 5. A J-shaped transition curve in normalized form.

Above discussion concludes the following.

$$
\begin{gathered}
\boldsymbol{p}_{0}=(0,0), \quad \boldsymbol{p}_{1}=(g, 0), \\
\boldsymbol{p}_{2}=\boldsymbol{p}_{1}+(h, 0), \quad \boldsymbol{p}_{3}=\boldsymbol{p}_{2}+k(\cos 2 \theta, \sin 2 \theta) .
\end{gathered}
$$

Since control points $\boldsymbol{p}_{0}, \boldsymbol{p}_{1}$, and $\boldsymbol{p}_{2}$ are collinear and distinct, we can assume $\boldsymbol{p}_{1}$ is lying on a parametric straight line with its end-points at $\boldsymbol{p}_{0}$ and $\boldsymbol{p}_{2}$, i.e., $\boldsymbol{p}_{1}=(1-m) \boldsymbol{p}_{0}+m \boldsymbol{p}_{2}$, where parameter $m \in(0,1)$. Here the end-points curvature conditions are $(\kappa(0), \kappa(1))=\left(0,1 / r_{1}\right)$. We also require osculating circle $\Omega_{1}$ with center $\boldsymbol{C}_{1}=\boldsymbol{z}(1)-r_{1}(\sin 2 \theta,-\cos 2 \theta)$ be tangent to the $x$-axis, i.e.,

$$
y(1)+r_{1} \cos 2 \theta=r_{1},
$$

leaving both $u$ and $m$ as free parameters. 
All the above mentioned conditions yield

$$
(g, h, k)=r_{1} \tan \theta\left(\frac{3 m \sec ^{2} \theta}{4(1-m)}, \frac{3 \sec ^{2} \theta}{4}, 1\right) .
$$

Then from (3.1), we have a family of cubic transition curves

$$
\begin{aligned}
x(t)= & \frac{r_{1} t \sqrt{u}}{4(1-m)(1+u)}\left[t\left\{9(1+u)^{2}-2 t\left(1+8 u+3 u^{2}\right)\right\}\right. \\
& \left.+m\left\{9(1+u)^{2}-18 t(1+u)^{2}+t^{2}\left(5+22 u+9 u^{2}\right)\right\}\right], \\
y(t)= & 2 r_{1} t^{3} u /(1+u) .
\end{aligned}
$$

To find sufficient conditions for the single curvature extremum in a J-shaped transition curve, the derivative of curvature of function (3.1) is required which is given by (2.2). It is calculated for $t=s /(1+s), s \geq 0$, and is represented as

$$
\phi(s)=\frac{243 r_{1}^{4} u^{5 / 2}}{16(1-m)^{3}(1+s)^{5}} \sum_{i=0}^{5} j_{i} s^{i},
$$

where the coefficients

$$
\begin{aligned}
& j_{0}=9 m^{3}(1+u)^{2}, \quad j_{1}=45 m^{3}(1+u)^{2}, \\
& j_{2}=6 m(1-m)\left\{-9(1+u)^{2}+m\left(31+86 u+39 u^{2}\right)\right\}, \\
& j_{3}=6(m-1)\left[6(1+u)^{2}+5 m^{2}\left\{1+26 u+9 u^{2}-m\left(19+134 u+51 u^{2}\right)\right\}\right], \\
& j_{4}=4(m-1)^{2}\left\{3-84 u-27 u^{2}-m\left(7-108 u-27 u^{2}\right)\right\}, \\
& j_{5}=8(1-m)^{2}\{1-9 u-m(3-9 u)\}
\end{aligned}
$$

are giving the sufficient condition for the single curvature extremum by Descartes rule of signs as $\min \left[-j_{5}, j_{3}, j_{2}, j_{1}, j_{0}\right] \geq 0$, i.e.,

$$
\frac{19-\sqrt{241}}{10}<m<\frac{11+\sqrt{73}}{20}, \quad u>0 .
$$

Remark 3. The distance between end-points $\boldsymbol{p}_{0}$ (i.e., origin) and $\boldsymbol{p}_{3}$ of transition curve is given by

$$
\|\boldsymbol{z}(1)\|=\frac{r_{1} \sec ^{2} \theta \tan \theta}{4(1-m)} \sqrt{\left\{3+4(1-m) \cos ^{2} \theta \cos 2 \theta\right\}^{2}+64 \cos ^{6} \theta \sin ^{2} \theta} .
$$

It is noticed that the transition curve reduces to the common point of the straight line and the circle as $\theta$ tends to 0 .

The following theorem guarantees the fairness of the desired single J-shaped transition curve for $(u, m)$ belonging to the gray region in Figure 6 or $u>0$ and $m \in[0.35,0.97]$.

Theorem 5. The $G^{2}$ transition curve $\boldsymbol{z}(t)$ of the form (3.4) defined by (3.1) with given angle $\theta$ and parameter $m$, between the straight line and the circle tangent to each other, exists and does not has more than one curvature extremum for conditions in (3.32).

Proof. Existence of solution follows immediately from the above discussion and the guarantee of single interior curvature extremum is given by (3.32). 


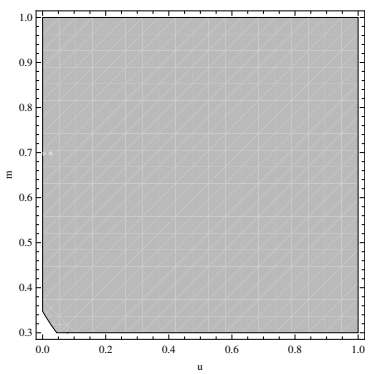

Figure 6. Regions for single curvature extremum for a J-shaped transition curve.

\section{The Algorithm}

Based on the above analysis, we have adopted the approach to construct a cubic Bézier spiral transition of C-, S-, and J-shaped, with a single polynomial in parametric form, for use in smoothing arc splines and spur gear designing of $G^{2}$ continuity. The procedure is described in the following algorithm.

1. Given are start point, tangent direction at start point, and curvatures at endpoints.

2. Normalize the given data for C-, S-, and J-shaped transition by transformation according to Figures 1, 3, and 5, respectively.

3. (a) For C-shaped transition, either select parameter $m$ from gray region in Figure 2(a) and find $u$ from (3.9) or select $u$ from gray region in Figure 2(b) and find $m$ from (3.13).

(b) For S-shaped transition, either select parameter $m$ from gray region in Figure 4(a) and find $u$ from (3.21) or select $u$ from gray region in Figure 4(b) and find $m$ from (3.26).

(c) For J-shaped transition, fix any positive value of $u$, and assign value of $m$ in the range $[0.35,0.97]$, or select $(u, m)$ from gray region in Figure 6.

4. Find $\theta=\arctan \sqrt{u}$.

5. Find the control points from (3.2), (3.15), and (3.27) for C-, S-, and J-shaped, respectively.

6. Construct Bézier transition curve from (3.1).

7. View the curvature plot for this curve. Continue, if it is acceptable, otherwise, go to step 3 and try some other value of $m$ (or $\theta$ ).

8. Apply the reverse transformation to bring the transition curve back to the pre-normalized position of its given data. 


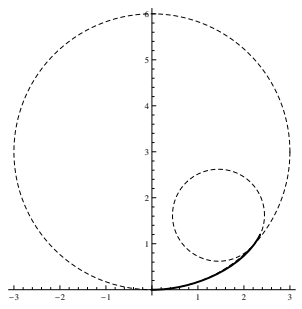

(a) Case 1: $(\mu, m)=(1.732,0.875)$

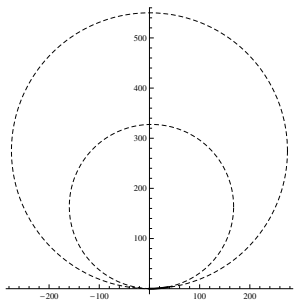

(d) Case 2: $(\mu, u)=(1.296,0.01)$

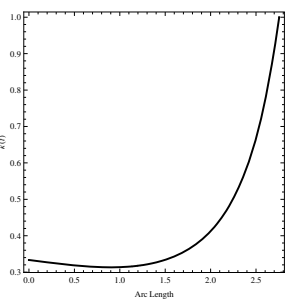

(b) The Curvature plot

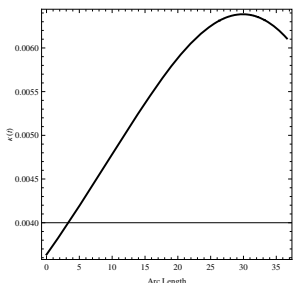

(e) The Curvature plot

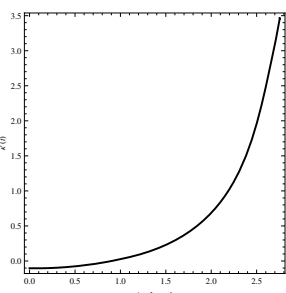

(c) Derivative of curvature

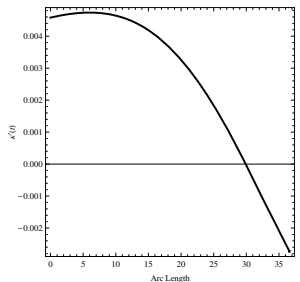

(f) Derivative of curvature

Figure 7. C-shaped transition curves (left) with single curvature extremum.

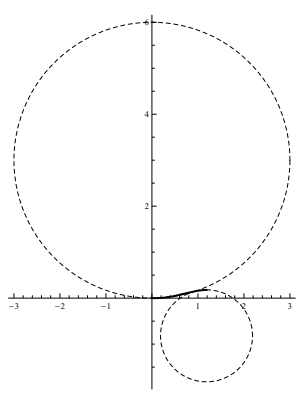

(a) Case 1: $(\mu, m)=(1.732,0.6)$

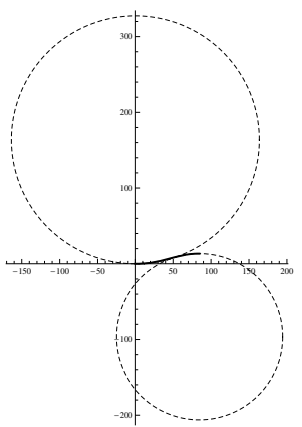

(d) Case 2: $(\mu, u)=(1.221,0.8)$

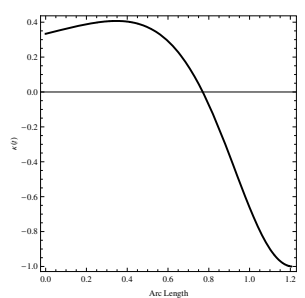

(b) The Curvature plot

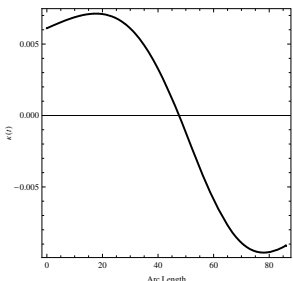

(e) The Curvature plot

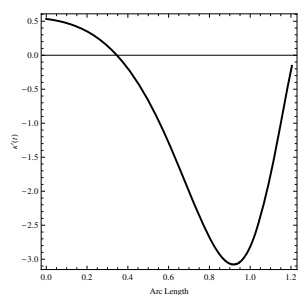

(c) Derivative of curvature

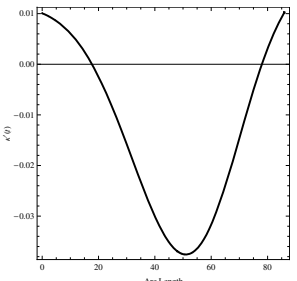

(f) Derivative of curvature

Figure 8. S-shaped transition curves (left) with single (upper) and double (down) curvature extrema. 


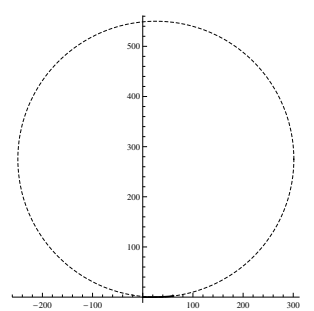

(a) $\left(r_{1}, u, m\right)=(275.02,0.004,0.7)$

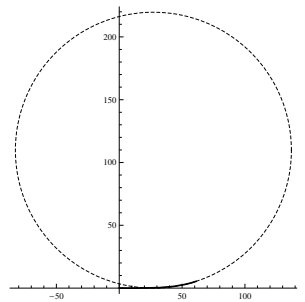

(d) $\left(r_{1}, u, m\right)=(109.81,0.025,0.7)$

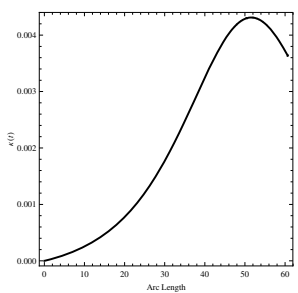

(b) The Curvature plot

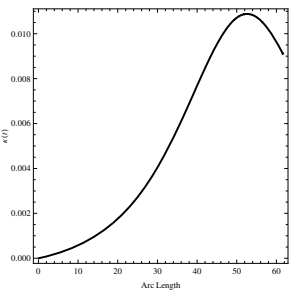

(e) The Curvature plot

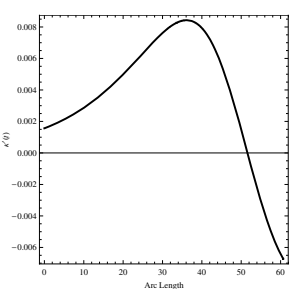

(c) Derivative of curvature

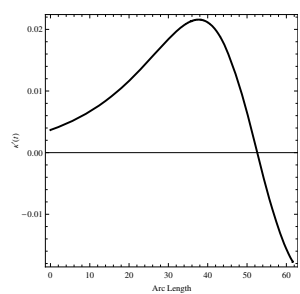

(f) Derivative of curvature

Figure 9. J-shaped transition curves (left) with single curvature extremum.

\section{Fairing Arc Spline}

For the use of cubic Bézier spiral transitions of C-, S-, and J-shaped in smoothing arc spline with $G^{2}$ continuity, first we consider the following numerical examples of all cases of transition curves with their corresponding curvature plots and derivative of curvature plots. Data of these examples is in normalized form. Locations of selected free parameters are shown with white stars in Figures 2, 4, and 6 .

Example 1. C-shaped transitions

Case 1: Given parameters are $(\mu, m)=(1.732,0.875)$ and parameter $u$ is calculated. Since $(\mu, m)$ belongs to the gray region in Figure 2(a), therefore the transition curve in Figure 7 (a) has just a single curvature extremum.

Case 2: Given parameters $(\mu, u)=(1.296,0.01)$ are selected from the gray region in Figure 2(b) and parameter $m$ is calculated. The transition curve in Figure $7(\mathrm{~d})$ has just a single curvature extremum in this case also.

Example 2. S-shaped transitions

Case 1: Given parameters are $(\mu, m)=(1.732,0.6)$ and parameter $u$ is calculated. Since $(\mu, m)$ belongs to the light gray region in Figure 4(a), therefore the transition curve in Figure 8(a) has just a single curvature extremum.

Case 2: Given parameters are $(\mu, u)=(1.221,0.8)$ and parameter $m$ is calculated. Since $(\mu, u)$ belongs to the dark gray region in Figure 4(b), 
therefore the transition curve in Figure 8(d) has two curvature extrema in this case.

\section{Example 3. J-shaped transitions}

It does not has separate cases. However two different examples are considered for the use in our smoothed version of arc spline example. First example is with given parameters $\left(r_{1}, u, m\right)=(275.02,0.004,0.7)$ and second example is with $\left(r_{1}, u, m\right)=(109.81,0.025,0.7)$. Since in both examples $(u, m)$ belongs to the gray region in Figure 6 , therefore the transition curves in Figures $9(\mathrm{a})$ and $9(\mathrm{~d})$ have just a single curvature extremum.

Number of curvature extrema in all above examples of transition curves are verified in their corresponding curvature plots and derivative of curvature plots in Figures $7-9$.

\subsection{Smoothed arc spline}

An arc spline of Figure 6 in [20] is selected. It has five elements connected with $G^{1}$ continuity and its total arc length is 465 . Parts of these elements along both sides of their joints are replaced with J-, C-, S-, and again J-shaped spiral transition curves shown in Figures $9(\mathrm{a}), 7(\mathrm{~d}), 8(\mathrm{~d})$, and $9(\mathrm{~d})$, respectively. Endpoints of arc spline elements are shown with small gray disks, replaced parts are highlighted with black in smoothed version of arc spline, and the remaining parts are shown with gray in Figure 10(a). All the segments are connected with $G^{2}$ continuity. The total arc length of this smoothed version is 464.9 and its curvature plot is given in Figure 10(b).

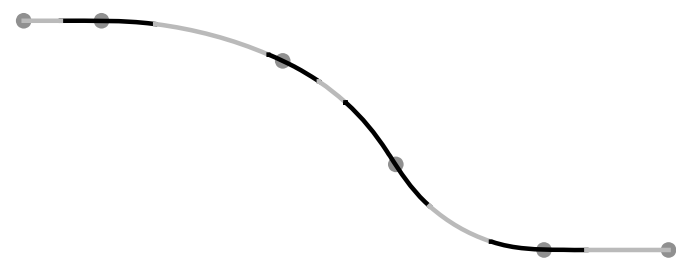

(a) A spiral spline

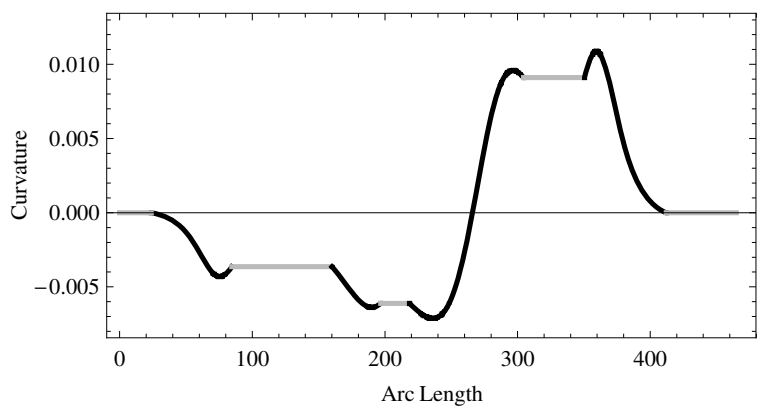

(b) The curvature plot of spiral spline

Figure 10. A smoothed version of the arc spline of Fig. 6 in [20]. 


\section{Geometric Designing}

Transition curve used in fairing arc spline can conveniently be used for modeling spur gear teeth. Gear teeth are the fundamental parts of engineering and automobile. Involutes, evolutes, transcendental functions, and B-spline functions are being used for the geometric design of a gear tooth [1, 4, 8, 34]. All these methods are without any consideration or proof of number of internal curvature extrema in the used transition curve. Degree of smoothness of a tooth profile can be increased by minimizing the number of curvature extrema, resulting less friction and wear between two sliding surfaces. We have used an S-shaped transition curve between two circles of same radii and tangent outside to each other. As already proved in Corollary 1, this transition has minimum possible number of curvature extrema. Results are shown in Figure 11.

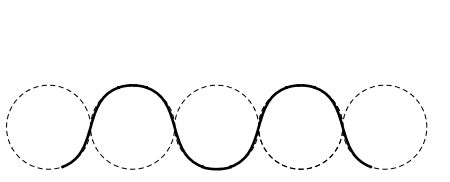

(a) Gear tooth

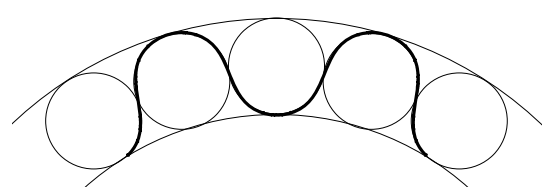

(b) Gear train

Figure 11. Gear design using S-shaped transition curve.

Comparison. Our main concern and focus is to approximate the arc-spline with the guarantee of minimum possible number of curvature extrema by replacing their parts using single cubic Bézier curve while keeping the level of smoothness to $G^{2}$ continuity. Transition curves can always be made shorter so that they are closer to the arc spline. Therefore, little or much of the original arc spline can be retained by using the available degree of freedom. The comparison of the above results with those in [20] is summarized in Table 1.

Table 1. Comparison of our results with the results in [20].

\begin{tabular}{|c|c|c|}
\hline No. & Results in [20] & Our results \\
\hline 1 & $\begin{array}{l}\text { A pair of conics was used to smooth } \\
\text { a C-shaped curve and a cubic Bézier } \\
\text { curve was used to smooth S- and } \\
\text { J-shaped curves. }\end{array}$ & $\begin{array}{l}\text { A single cubic Bézier curve for all } \\
\text { types of transition curves is used. }\end{array}$ \\
\hline 2 & $\begin{array}{l}\text { S-, C- and J-shaped transition com- } \\
\text { prise three to five, three to six, and } \\
\text { two to five spiral pieces, respectively. }\end{array}$ & $\begin{array}{l}\text { S-shaped transition comprises two to } \\
\text { three spiral pieces. C- and J-shaped } \\
\text { transition comprise exactly two spi- } \\
\text { ral pieces. }\end{array}$ \\
\hline 3 & $\begin{array}{l}\text { Conditions are not provided for the } \\
\text { number of curvature extrema. }\end{array}$ & $\begin{array}{l}\text { Sufficient conditions are provided for } \\
\text { the exact number of curvature ex- } \\
\text { trema. }\end{array}$ \\
\hline
\end{tabular}




\section{Conclusion and Future Research Work}

We presented a method for smoothing a $G^{1}$ arc spline to $G^{2}$ continuity by replacing its three types of parts (C-, S-, and J-shaped) with spiral transition curves. The main advantages of our method are that all kinds of transition curves can be achieved from a single cubic Bézier curve of fixed and low degree providing designers and implementers fewer entities to be concerned. Curves are comprising of minimum possible number of internal curvature extrema.

A PH (Pythagorean Hodograph) quintic curve has the attractive properties that its arc length is a polynomial of its parameter, and its offset is rational. A quintic is the lowest degree $\mathrm{PH}$ curve that may has an inflection point. These curves have been discussed in [11, 12, 23, 24,31] providing more flexible constraints and more wider range of free parameters. Therefore the smoothing process of arc spline and gear designing can also be tried with $\mathrm{PH}$ quintic transition curves.

The proposed technique for spur gear geometry representation can be extended to various gear typologies, e.g., helical, bevel, hypoid, crown, etc. Future work should be directed towards the development of simple and refined techniques for geometric modeling of gears for the purpose of surface generation based on constraining procedures.

\section{Acknowledgment}

The authors owe their gratitude to the four anonymous referees for their valuable comments and suggestions which have helped to improve the presentation of the paper and quality of research. We would also like to thank Aisha Rashid and Iffat Fayyaz for the implementation of some results of this manuscript.

\section{References}

[1] S. Barone. Gear geometric design by B-spline curve fitting and sweep surface modelling. Engineering with Computers, 17:66-74, 2001. http://dx.doi.org/10.1007/s003660170024.

[2] X.-D. Chen, J.-H. Yong, G.-Q. Zheng and J.-G. Sun. Automatic $g^{1}$ arc spline interpolation for closed point set. Computer-Aided Design, 36(12):1205-1218, 2004. http://dx.doi.org/10.1016/j.cad.2003.12.001.

[3] S. Dimulyo, Z. Habib and M. Sakai. Fair cubic transition between two circles with one circle inside or tangent to the other. Numer. Algorithms, 51(4):461-476, 2009. http://dx.doi.org/10.1007/s11075-008-9252-1.

[4] D.W. Dudley. Handbook of Practical Gear Design. CRC Press, 1994.

[5] G. Farin. NURB Curves and Surfaces. A K Peters, 2nd edition, 1999.

[6] G. Farin. Curves and Surfaces for CAGD: A Practical Guide. MorganKaufmann, 5th edition, 2002.

[7] G. Farin. Geometric Hermite interpolation with circular precision. ComputerAided Design, 40(4):476-479, 2008.

http://dx.doi.org/10.1016/j.cad.2008.01.003. 
[8] I. Fayyaz, A. Rashid and Z. Habib. Spur gear tooth designing with an S-shaped cubic Bézier transition curve. In International Conference on Intelligence and Information Technology, Lahore, 2010. Presented

[9] H. Guggenheimer. Differential Geometry. McGraw-Hill, New York, 1963.

[10] Z. Habib. Spiral Function and Its Application in CAGD. VDM Verlag, Germany, 2010. ISBN: 978-3-639-24988-0

[11] Z. Habib and M. Sakai. $G^{2}$ Pythagorean hodograph quintic transition between two circles with shape control. Comput. Aided Geom. Design, 24(5):252-266, 2007. http://dx.doi.org/10.1016/j.cagd.2007.03.004.

[12] Z. Habib and M. Sakai. On PH quintic spirals joining two circles with one circle inside the other. Computer-Aided Design, 39(2):125-132, 2007. http://dx.doi.org/10.1016/j.cad.2006.10.006.

[13] Z. Habib and M. Sakai. $G^{2}$ cubic transition between two circles with shape control. Comput. Appl. Math., 223:133-144, 2009. http://dx.doi.org/10.1016/j.cam.2007.12.024.

[14] Z. Habib and M. Sakai. Smoothing arc splines by cubic curves. In The Proceedings of IEEE International Conference on Computer Graphics, Imaging and Visualization, China, pp. 199-204, USA, 2009. IEEE Computer Society Press.

[15] Z. Habib and M. Sakai. Admissible regions for rational cubic spirals matching $G^{2}$ Hermite data. Computer-Aided Design, 42(12):1117-1124, 2010. http://dx.doi.org/10.1016/j.cad.2010.07.006.

[16] Z. Habib, M. Sarfraz and M. Sakai. Rational cubic spline interpolation with shape control. Computers \& Graphics, 29(4):594-605, 2005. http://dx.doi.org/10.1016/j.cag.2005.05.010.

[17] P. Henrici. Applied and Computational Complex Analysis, volume 1. Wiley, New York, 1988.

[18] J. Hoschek. Circular splines. Computer-Aided Design, 24:611-618, 1992. http://dx.doi.org/10.1016/0010-4485(92)90072-I.

[19] J. Hoschek and D. Lasser. Fundamentals of Computer Aided Geometric Design (Translation by L.L. Schumaker). A. K. Peters, Wellesley, MA, 1993.

[20] Z. Li and D.S. Meek. Smoothing an arc spline. Computers \& Graphics, 29:576587, 2005. http://dx.doi.org/10.1016/j.cag.2005.05.009.

[21] Z. Li, D.S. Meek and D.J. Walton. A smooth, obstacle-avoiding curve. Computers E Graphics, 30(4):581-587, 2006. http://dx.doi.org/10.1016/j.cag.2006.03.003.

[22] D.S. Meek, B.H. Ong and D.J. Walton. A constrained guided $G^{1}$ continuous spline curve. Computer-Aided Design, 35:591-599, 2003. http://dx.doi.org/10.1016/S0010-4485(02)00086-6.

[23] A. Rashid, I. Fayyaz and Z. Habib. Using PH quintic transition curves in gear tooth designing. In International Conference on Intelligence and Information Technology, Lahore, 2010. Presented

[24] A. Rashid, I. Fayyaz, Z. Habib and M. Sakai. Smoothing an arc spline using $\mathrm{PH}$ quintic spiral transitions. In International Conference on Intelligence and Information Technology, Lahore, 2010. Presented

[25] M. Sakai. Inflection points and singularities on planar rational cubic curve segments. Comput. Aided Geom. Design, 16(3):149-156, 1999.

http://dx.doi.org/10.1016/S0167-8396(98)00036-3. 
[26] M. Sakai. Osculatory interpolation. Comput. Aided Geom. Design, 18(8):739750, 2001. http://dx.doi.org/10.1016/S0167-8396(01)00052-8.

[27] M. Sarfraz(Ed.). Advances in Geometric Modeling. Wiley, England, 2003.

[28] M. Sarfraz(Ed.). Geometric Modeling: Techniques, Applications, Systems and Tools. Kluwer, Netherlands, 2004.

[29] D.J. Walton and D.S. Meek. Planar $G^{2}$ transition between two circles with a fair cubic Bézier curve. Computer-Aided Design, 31(14):857-866, 1999. http://dx.doi.org/10.1016/S0010-4485(99)00073-1.

[30] D.J. Walton and D.S. Meek. Curvature extrema of planar parametric polynomial cubic curves. J. Comput. Appl. Math., 134(1-2):69-83, 2001. http://dx.doi.org/10.1016/S0377-0427(00)00529-X.

[31] D.J. Walton and D.S. Meek. $G^{2}$ curve design with a pair of Pythagorean hodograph quintic spiral segments. Comput. Aided Geom. Design, 24(5):267-285, 2007. http://dx.doi.org/10.1016/j.cagd.2007.03.003.

[32] D.J. Walton and D.S. Meek. $G^{2}$ Hermite interpolation with circular precision. Computer-Aided Design, 42(5):749-758, 2010. http://dx.doi.org/10.1016/j.cad.2010.04.004.

[33] D.J. Walton, D.S. Meek and J.M. Ali. Planar $G^{2}$ transition curves composed of cubic Bézier spiral segments. Comput. Appl. Math., 157(2):453-476, 2003. http://dx.doi.org/10.1016/S0377-0427(03)00435-7.

[34] S.H. Yahaya, M.S. Salleh and M.A. Jamaludin. Spur gear design with an sshaped transition curve application using MATHEMATICA and CAD tools. In The Proceedings of IEEE International Conference on Computer Technology and Development, pp. 426-429, USA, 2009. IEEE Computer Society Press. http://doi.ieeecomputersociety.org/10.1109/ICCTD.2009.76

[35] J.-H. Yong, X. Chen and J.-C. Paul. An example on approximation by fat arcs and fat biarcs. Computer-Aided Design, 38(5):515-517, 2006. http://dx.doi.org/10.1016/j.cad.2006.01.003.

[36] J.-H. Yong and F. Cheng. Geometric Hermite curves with minimum strain energy. Comput. Aided Geom. Design, 21(3):281-301, 2004. http://dx.doi.org/10.1016/j.cagd.2003.08.003.

[37] J.-H. Yong, S.-M. Hu and J.-G. Sun. A note on approximation of discrete data by $G^{1}$ arc splines. Computer-Aided Design, 31(14):911-915, 1999. http://dx.doi.org/10.1016/S0010-4485(99)00076-7.

[38] J.-H. Yong, S.-M. Hu and J.-G. Sun. Bisection algorithms for approximating quadratic Bézier curves by $G^{1}$ arc splines. Computer-Aided Design, 32(4):253260, 2000. http://dx.doi.org/10.1016/S0010-4485(99)00100-1. 